\title{
Bioequivalence of Topical Dosage Forms
}

\section{Piyush Jain*}

Formulation Scientist, GlaxoSmithKline, USA

Skin being the largest organ of the human body and because it offers specific advantages over other routes such as ease of access and bypassing the first pass metabolism, it presents a tremendous opportunity for administering drugs. Dosage forms meant to deliver drugs via skin can be broadly classified into topical and transdermal dosage forms. Topical dosage forms such as gels, creams, ointments, lotions are meant to be applied on skin for localized delivery of drugs to the affected areas. They differ from transdermal dosage forms, such as patches, in the fact that the drugs applied topically are less likely to be absorbed into the systemic circulation. Their site of action is either in one of the skin layers or in deeper tissues under the skin. The differences in composition and method of fabrication can be key aspects which dictate the site of delivery of drug from these dosage forms. The blood supply, however being present in the dermal layer of skin, can lead to some absorption of drugs to the systemic circulation even from topical dosage forms.

Manufacture and sale of any drug product are governed by federal laws of regulation. The innovator company gets the sole rights and permission to manufacture and sell a new drug product for a certain period of time. Once that patent for the drug product expires, any company other than the innovator company can manufacture and sell that drug product. For any dosage form however, a generic company has to submit bioequivalence data in order to show equivalency to the innovator product. According to Federal Drug and Cosmetic Act, section 505 ( $\mathrm{j}$ ) (8) (B), bioequivalence for drug is defined as:

"A drug shall be considered bioequivalent to a listed drug if the rate and extent of absorption of the drug do not show any significant difference from the rate and extent of absorption of the listed drug when administered at the same molar dose of the therapeutic ingredient under similar experimental conditions in a single dose or multiple doses".

For dosage forms which show their efficacy by getting absorbed into the systemic circulation (such as patches), blood levels of drug are a good measure of rate and extent of absorption of drug as it appropriately reflects the amount of drug at its site of action. For topical dosage forms, however, the blood levels might not be even detectable or the drug might have multiple routes, making blood levels not representative of the amount of drug present at the site of action. For such dosage forms, according to Federal Drug and Cosmetic Act, section 505 (j) (8) (C):

"For a drug that is not intended to be absorbed into the blood stream, the secretary may establish alternative, scientifically valid methods to show bioequivalence if the alternative methods are expected to detect a significant difference between the drug and the listed drug in safety and therapeutic effect".

Normally clinical efficacy trials are considered as gold standard for showing bioequivalence for such dosage forms. The studies are however insensitive, costly, time consuming and require large number of subjects. There are a few alternate methods which have been proposed to show equivalency for topical dosage forms. Brief detail and status of their acceptance is as below:

\section{Skin Blanching Assay}

It is a pharmacodynamic method which is accepted by FDA to show equivalency of topical dosage forms containing glucocorticoids. The site of action of glucocorticoids is the glucocorticoid receptors in the viable epidermis and dermis. The pharmacodynamic response which is measured is the constriction of microvasculature of skin, which causes skin blanching (whitening) at the site of action.

\section{Pharmacokinetic Method (PK)}

As mentioned earlier, blood levels are not an adequate measure of the drug present at the site of action for topical dosage forms. There is just one specific case where the agency accepts PK data to show bioequivalence. Lidocaine patches $5 \%$ are meant to be applied on skin for local anesthesia. In this case, sufficient drug reaches the blood circulation to be detected and drug level in plasma is proportional to the amount of drug at the site of action (nerves in dermal tissue). PK data can therefore be submitted while showing bioequivalence for Lidocaine patches $5 \%$.

\section{Tape Stripping/ Dermatopharmacokinetic (DPK) Method}

In this method, layers of stratum corneum are sequentially removed and drug concentration in the stratum corneum is measured as a function of time. The stratum corneum concentration - time profiles for two products can then be compared for bioequivalence. The rationale behind this is that topically applied drugs must pass through stratum corneum before reaching into deeper layers analogous to orally administered drugs which have to pass blood circulation before reaching the site of action. This method was under serious consideration by FDA and a draft guidance was issued in 1998. This guidance was however withdrawn later in May, 2002 when contradictory results were obtained from two independent laboratories using this method. Currently, efforts are needed in this area to standardize the protocol for tape stripping process and revalidate the method.

\section{In-vitro Permeation Method}

The method involves testing permeation of drug across excised human/ animal skin using apparatus similar to Franz cells. Good invitro in-vivo correlation data has been shown by a couple of researchers using this method. The agency however does not accept it as a surrogate for bioequivalence. The limitations to this model include lack of live tissue, underlying supportive structure, metabolic activity and systemic circulation.

\section{In-vitro Release Method}

This method is similar to in-vitro permeation but uses a synthetic

${ }^{*}$ Corresponding author: Piyush Jain, Formulation Scientist, GlaxoSmithKline, GSK 1500 Littleton Road Parsippany NJ 07054, USA, Tel: +1 2175020587 ; E-mail: piyush.2.jain@gsk.com

Received February 27, 2014; Accepted February 28, 2014; Published March 08, 2014

Citation: Jain P (2014) Bioequivalence of Topical Dosage Forms. J Pharmacovigilance 2: e118. doi:10.4172/2329-6887.1000e118

Copyright: (c) 2014 Jain P. This is an open-access article distributed under the terms of the Creative Commons Attribution License, which permits unrestricted use, distribution, and reproduction in any medium, provided the original author and source are credited. 
membrane instead of skin to assess the release of drug from formulations without incorporating barrier properties of skin. This method does not act as surrogate for clinical studies but can be used as a useful test to assess product sameness under certain scale up and post approval changes (SUPAC). There is a new chapter (chapter no 1724) which has recently been added in year 2013 to the USP 36-NF 31, detailing the in-vitro release method and its application.

There is another approach which one can take to show bioequivalency, which is based on pharmaceutical equivalence. There are three stages of similarity which the generic company has to show between their product and the innovator product. These can be classified as follows:

Q1 similar: Generic product has same ingredients as the innovator product

Q2 similar: Same ingredients in same concentration

Q3 similar: Same ingredients in same concentration in same microstructure.

Given that the innovator companies do not disclose all of their ingredients and their concentrations, it becomes difficult for the generic companies to reverse engineer their product and make it Q1/ Q2 similar to the innovator product. In addition, the microstructure of a dosage form may vary according to the processing conditions during manufacture. As such, there is no appropriate guidance or techniques which can ensure Q3 similarity between the products. There are however certain exceptions where the regulatory body accepts applications without showing clinical efficacy data. These are as follows:

\section{Acyclovir Ointment 5\%}

The agencies accept applications for products which are Q1/Q2 similar and show Q3 equivalency using in-vitro release. A reason for this particular case might be that the ointment has very low potency and an end point clinical study may not be feasible or reliable for such a case.

\section{Topical Solutions}

A biowaiver may be granted for such products if the generic product contains same active ingredient in the same concentration as the innovator product and has no inactive ingredient (such as permeation enhancer) or other change which affects bioavailability.

\section{Products Coded “AT” in Orange Book}

These products refer to few dosage forms approved before year 1962. A biowaiver can be granted for such products if the generic and innovator product have same ingredients in same dosage form. Specific examples for this can be erythromycin topical gel, hydrocortisone topical cream and likewise.

\section{Summary}

Bioequivalence for topical dosage forms is currently limited to clinical efficacy trials where the outcome is dichotomous in nature in the form of "Yes" or "No". This results in very low statistical significance of these trials and requires several hundreds of subjects to be enrolled. Considering this, FDA also acknowledges the need to develop surrogate methods to show bioequivalence of topical drug products. This is however challenging given the number of sites of action for different topical products and just a fraction of dose (generally less than 1\%) being getting absorbed through skin. Method optimization followed by validation and verification at multiple laboratories and above all commitment from industries and agency to provide infrastructure and resources is the need of hour. 